\title{
The gut microbiota contributes to the development of Staphylococcus aureus-induced mastitis in mice
}

\author{
Xiaoyu Hu${ }^{1} \cdot$ Jian Guo ${ }^{1}$ Caijun Zhao ${ }^{1} \cdot$ Peng Jiang $^{1} \cdot$ T. Maimai ${ }^{1} \cdot$ Li Yanyi $^{1} \cdot$ Yongguo Cao $^{1} \cdot$ Yunhe Fu$^{1}$. \\ Naisheng Zhang ${ }^{1}$
}

Received: 11 March 2019 / Revised: 27 March 2020 / Accepted: 31 March 2020 / Published online: 27 April 2020

(c) The Author(s), under exclusive licence to International Society for Microbial Ecology 2020

\begin{abstract}
Mastitis is one of the most prevalent diseases in dairy farming worldwide. The gut microbiota plays an important role in the regulation of systemic and local inflammatory diseases, such as mastitis. However, the regulatory mechanism of the gut microbiota on mastitis is still unclear. Thus, the aim of this study was to investigate the function and regulatory mechanisms of the gut microbiota in host defense against mastitis caused by Staphylococcus aureus (S. aureus) infection. Increased blood-milk barrier permeability, and $S$. aureus-induced mastitis severity were observed gut microbiota-dysbiosis mice compared with those in control mice. Moreover, feces microbiota transplantation (FMT) to microbbiota-dysbiosis mice reversed these effects. Furthermore, established disruption of commensal homeostasis results in significantly increased abundance of pathogenic Enterobacter bacteria, while the relative abundance of short-chain fatty acid (SCFAs)-producing bacterial phyla (Firmicutes and Bacteroidetes) was significantly reduced. However, FMT to gut microbiota-dysbiosis mice reversed these changes. In addition, dysbiosis reduced the levels of SCFAs, and administration of sodium propionate, sodium butyrate, and probiotics (butyrate-producing bacteria) reversed the changes in the blood-milk barrier and reduced the severity of mastitis induced by $S$. aureus. In conclusion, this new finding demonstrated that the gut microbiota acts as a protective factor in host defense against mastitis and that targeting the gut-mammary gland axis represents a promising therapeutic approach for mastitis treatment.
\end{abstract}

\section{Introduction}

Mastitis is one of the most important diseases in dairy farming. Mastitis decreases milk quality and quantity, which results in considerable economic losses worldwide $[1,2]$. Staphylococcus aureus (S. aureus) is frequently isolated from subclinical mastitis and is one of the most commonly diagnosed bacteria in animals suffering from this

Supplementary information The online version of this article (https:// doi.org/10.1038/s41396-020-0651-1) contains supplementary material, which is available to authorized users.

Yunhe Fu

fuyunhesky@sina.com

$\triangle$ Naisheng Zhang

zhangns@jlu.edu.cn

1 Department of Clinical Veterinary Medicine, College of Veterinary Medicine, Jilin University, Changchun 130062 Jilin, People's Republic of China disease $[3,4]$. In the past decade, antibiotics represent the major method of mastitis treatment. However, this treatment often leads to the presence of antibiotics residue and the development of bacterial drug resistance [5, 6]. Therefore, it is urgent to seek novel targets and drugs to treat mastitis.

In recent years, gut microbiota have attracted increasing attention. Gut microbiota play an important role in the regulation of health and disease [7]. Previous studies demonstrated that gut microbiota have been associated with the development of numbers diseases, such as diabetes, fatty liver, atherosclerosis, and cancer [8-13]. Recently, gut microbiota have been reported to be involved in infectious diseases, including mastitis [14]. A previous study demonstrated that the feces microbial community changes in mastitis cows were similar to those in milk, characterized by a general increase in Enterococcus, Streptococcus, and Staphylococcus abundances but deprivation of Lactobacillus [14]. Furthermore, some mammary gland bacteria derived from the gut by an endogenous entero-mammary pathway have been reported [15]. In addition, recent evidence suggests that feces microbiota transplantation (FMT) 
from mastitis cows but not from healthy cows to germ-free mice leads to mastitis symptoms in the mammary gland [16]. However, whether a relationship exists between the gut microbiota and $S$. aureus-induced mastitis and the relevant mechanism remain unclear.

Neutrophils (PMNs) are recognized as the major diagnostic indictor of bovine mastitis. Upon stimulation by invading pathogens, PMNs migrate from the blood through the basement membrane and mammary epithelium into the milk [17]. The blood-milk barrier plays an important role in the transfer of PMNs from blood to milk. Increased permeability of the blood-milk barrier leads to a large number of PMNs in the mammary gland [18]. The gut microbiota plays a crucial role in maintaining bloodtissue barriers, including the blood-brain barrier (BBB) and blood-testis barrier (BTB). BBB and BTB permeability were increased in germ-free mice $[19,20]$. In this study, we hypothesized that alterations of the gut microbiota alter blood-milk barrier permeability and alter the threshold of PMNs crossing the blood-milk barrier. Thus, numbers of PMNs enter in the mammary gland and aggravate mastitis.

\section{Materials and methods}

\section{Animals}

BALB/c mice (336 female and 168 male), at 8-12 weeks old were purchased from the Center of Experimental Animals of Baiqiuen Medical College of Jilin University. One male and two females were housed together in one microisolator cage. SPF grade mice breeding fodder and water were supplied ad libitum. When pregnancy was confirmed, each female was housed individually and subjected to subsequent experiments.

\section{Materials}

Staphylococcus aureus SA113 (ATCC35556) was obtained from Mikrobielle Genetik, University of Tubingen, Germany. Clostridium tyrobutyricum (C. tyroburyricum, ATCC25755) was purchased from ATCC. Sodium butyrate, sodium propionate, sodium acetate, and FITC-albumin were purchased from Sigma (St. Louis, MO, USA). Occludin, Claudin-3, and ZO-1 antibodies were purchased from Cell Signaling Technology, Inc. (Beverly, MA). Alexa Fluor 488 anti-mouse Ly-6G/Ly-6C (GR-1), FITC Rat Anti-mouse F4/80, PE Rat Anti-CD11b, PerCP-Cy ${ }^{\mathrm{TM}} 5.5$ Hamster AntiMouse CD3, PE Rat Anti-Mouse CD8a, FITC Rat AntiMouse CD4, PE Rat Anti-Mouse CD49b, FITC Anti-mouse CD3 were purchased from Biolegend (USA). All other chemicals were of reagent grade.

\section{Abx (cocktail of antibiotics) treatment}

The mice were treated with broad spectrum antibiotics (ampicillin $1 \mathrm{~g} / \mathrm{L}$, Sigma: neomycin sulfate $1 \mathrm{~g} / \mathrm{L}$, Sigma: metronidazole $1 \mathrm{~g} / \mathrm{L}$, Sigma: and vancomycin $0.5 \mathrm{~g} / \mathrm{L}$, Scientific Research Ievei) in drinking water for 3 weeks. Antibiotics were removed from drinking water for 2 days, and then the mice were infected with $S$. aureus.

\section{Feces microbiota transplantation}

The process of feces microbiota transplantation was performed as previously described [21, 22]. Fresh feces were collected from untreated donor mice. Fecesl pellets from different healthy mice were mixed with sterile normal PBS (1 feces pellet $/ 1 \mathrm{~mL}$ of PBS) and homogenized immediately. The homogenate was centrifuged $\left(100 \times g, 5 \mathrm{~min}, 4^{\circ} \mathrm{C}\right)$, and the supernatant was collected for transplantation. A total of $200 \mu \mathrm{L}$ of the supernatant was administered to Abx treatment mice by oral gavage for 7 consecutive days. The mice of the control group were administered an equal volume of PBS.

\section{Establishment of mouse mastitis model}

The mouse mastitis model was induced by $S$. aureus as previously described [23]. Briefly, the offspring were removed $3 \mathrm{~h}$ before administration. Mice were anesthetized and placed on their back under a binocular. The teats and surrounding areas were disinfected with $70 \%$ ethanol. The udder canals of the fourth pair of mammary glands were held lightly with fine forceps and exposed. S. aureus dissolved in $50 \mu \mathrm{L}$ PBS was injected through the mammary gland ducts using a $100-\mu \mathrm{L}$ syringe with a 30-gauge blunt needle. Twenty-four hours later, the mice were sacrificed, and mammary glands were collected and stored at $-80^{\circ} \mathrm{C}$ until use.

\section{Treatment}

In the study of protective effect of SCFAs on mastitis infected by $S$. aureus, sodium butyrate, sodium propionic or sodium acetate (diluted in PBS, $100 \mathrm{mg} / \mathrm{kg}$ ) intraperitoneally was administered $1 \mathrm{~h}$ before $S$. aureus infection $\left(1 \times 10^{7} \mathrm{CFU} / 50 \mu \mathrm{L}\right.$ PBS $)$.

In the study of protective effects of clostridium tyrobutyricum (C. tyrobutyricum) on mastitis caused by $S$. aureus infection, C. tyrobutyricum $\left(1 \times 10^{6}, 1 \times 10^{7}\right.$, or $1 \times$ $10^{8} \mathrm{CFU} / 200 \mu \mathrm{L}$ PBS) were administered to mice by oral gavage for 7 consecutive days (once a day) before $S$. aureus $\left(1 \times 10^{7} \mathrm{CFU} / 50 \mu \mathrm{L} \mathrm{PBS}\right)$ infection.

In the study of the molecular mechanism of $C$. tyrobutyricum on mastitis, $C$. tyrobutyricum were cultured in supplemented medium for $8 \mathrm{~h}$, and the culture broth was centrifuged at $5000 \times g$ for $10 \mathrm{~min}$. The $C$. tyrobutyricum were suspended 
in sterile PBS, heat-inactivated $\left(120^{\circ} \mathrm{C}\right.$ for $\left.30 \mathrm{~min}\right)$ and resuspended with sterile PBS at a final concentration of $1 \times$ $10^{8} \mathrm{CFU} / 200 \mu \mathrm{L}$ to obtain inactive $C$. tyrobutyricum. In addition, spent culture supernatant of $C$. tyrobutyricum was collected and sterilized with a $0.22 \mu \mathrm{m}$ filter. Untreated mice were orally gavaged with PBS, heat-inactivated $C$. tyrobutyricum $\left(1 \times 10^{8} \mathrm{CFU} / 200 \mu \mathrm{L} \mathrm{PBS}\right)$, or $C$. tyrobutyricum supernatant $\left(200 \mu \mathrm{L}\right.$ of bacterial suspensions with $1 \times 10^{8} C$. tyrobutyricum) for 7 consecutive days (once a day) before $S$. aureus $\left(1 \times 10^{7} \mathrm{CFU} / 50 \mu \mathrm{L} \mathrm{PBS}\right)$ infection.

\section{Histopathologic assay and scoring of mammary gland tissue}

The mammary gland tissues were collected and immediately fixed in $10 \%$ formalin. The tissues were embedded in paraffin wax and subsequently dehydrated in a series of graded alcohols for hematoxylin and eosin staining. The scoring of histopathologic changes was performed as previously described [24], and was showed in Table S1.

\section{Immunohistochemical assay}

Mammary gland tissues were embedded in paraffin wax and sectioned. After dehydration in a graded series of alcohol solutions, the sections were incubated with a peroxidaseblocking solution for $30 \mathrm{~min}$. The sections were incubated with goat serum for $30 \mathrm{~min}$ and then incubated with an antimouse myeloperoxidase primary antibody at $4{ }^{\circ} \mathrm{C}$ overnight. The sections were incubated with a secondary antibody for $10 \mathrm{~min}$ and were incubated with a streptavidin anti-biotin peroxidase solution. Ten minutes later, the sections were incubated with a diaminobenzidine solution for 10 min. After staining with hematoxylin, the sections were observed under a light microscope.

\section{Flow cytometry}

The mammary glands from each group of mice were harvested, weighed, cut into pieces and digested with collagenase D (Sigma) for 1 hour at $37^{\circ} \mathrm{C}$. The homogenate was filtered with a $70 \mu \mathrm{m}$ filter, and cells were collected after centrifugation. Neutrophils, macrophages, T cells, and NK cells were identified using the following antibodies: anti-mouse GR-1, F4/80, and CD11b, CD3, CD4, CD8a, and CD49b. The data were analyzed with FlowJo (Treestar software, Ashland, OR, USA).

\section{Myeloperoxidase (MPO) activity assay}

MPO activity in the mammary gland tissues was tested using MPO kits according to the manufacturer's instructions (Jiancheng Bioengineering Institute of Nanjing, Nanjing,
China). The enzymatic activity was read based on absorbance at $460 \mathrm{~nm}$.

\section{Pro-inflammatory cytokine assay}

Tumor necrosis factor- $\alpha$ (TNF- $\alpha$ ), interleukin-1 $\beta$ (IL-1 $\beta$ ), and IL- 6 levels in the mammary gland tissues were assessed using enzyme-linked immune sorbent assay (ELISA) kits per the manufacturer's instructions (eBioscience, San Diego CA 92121 USA).

\section{FITC-albumin assay}

Blood-milk barrier permeability was assessed based on the expression of FITC-albumin as previously described $[25,26]$. In brief, mammary gland tissues were fixed in $10 \%$ formalin and then embedded in paraffin wax. The samples were sliced into $4 \mu \mathrm{m}$-thick sections, and the sections were dehydrated in a graded alcohols series. The sections were blocked with $5 \%$ goat serum for $1 \mathrm{~h}$ at $4{ }^{\circ} \mathrm{C}$, followed by incubation with FITC-albumin at $4{ }^{\circ} \mathrm{C}$ overnight. The sections were incubated with PBS containing $1 \%$ DAPI, and mounted with fluoromount.

\section{Western blot assay}

Total protein from mammary gland tissues was extracted using a tissue protein extraction reagent (Thermo). The protein concentration was detected using a BCA protein assay kit. The samples $(40 \mu \mathrm{g})$ were fractionated by $12 \%$ SDS-polyacrylamide gel and transferred to PVDF membranes. Membranes were blocked for $2 \mathrm{~h}$ with $3 \%$ BSA (dilution with TBS-T) at room temperature. Membranes were incubated with appropriate primary antibodies (dilution with TBS-T) at $4{ }^{\circ} \mathrm{C}$ overnight and then washed with TBS-T three times. The secondary antibody was incubated at room temperature for $1 \mathrm{~h}$ and then washed with TBS-T three times. The membranes were detected using the ECL Plus western blotting detection system. In addition, $\beta$-actin protein levels served as an internal control.

\section{Microbiota assay}

Fresh feces were collected under sterile conditions. Feces were immediately frozen in liquid nitrogen and then stored at $-80^{\circ} \mathrm{C}$. Total genome DNA samples were extracted using the CTAB/SDS method. $16 \mathrm{~S}$ rRNA genes of distinct regions were amplified used specific primers $(16 \mathrm{~S}$ V4:515F-806R) with barcodes. All PCR reactions were performed using Phusion ${ }^{\circledR}$ High-Fidelity PCR Master Mix (New England Biolabs). PCR products were purified using the Qiagen Gel Extraction Kit (Qiagen, Germany). Sequencing libraries were generated using the TruSeq ${ }^{\circledR}$ 
DNA PCR-Free Sample Preparation Kit (IIIumina, USA) following the manufacturer's recommendations and index codes were added. Library quality was assessed on the Qubit@ 2.0 Fluorometer (Thermo Scientific) and Agilent Bioanalyzer 2100 system. Finally, the library was sequenced on an IIIuminaHiSeq2500 platform, and $250 \mathrm{bp}$ paired-end reads were generated.

\section{SCFAs concentrations assay}

Fresh feces, blood, and mammary gland tissues were collected and immediately frozen and stored at $-80^{\circ} \mathrm{C}$. SCFAs concentrations in samples were assessed by gas chromatograph-mass spectrometer (Agilent 7890 A/5975 C, USA).

\section{Effect of sodium butyrate and sodium propionate on S. aureus growth}

To test the effect of sodium butyrate and sodium propionate on $S$. aureus growth, $1 \times 10^{7} \mathrm{CFU} / \mathrm{ml} \mathrm{S}$. aures was cultured at $37{ }^{\circ} \mathrm{C}$ in $\mathrm{LB}$ broth with different concentrations of butyrate and propionate $(1-5 \mathrm{mM})$ and growth was measured by the optical density at $600 \mathrm{~nm}$ for $24 \mathrm{~h}$.

\section{Determination of bacterial growth}

Blood was gained from the eyeballs of mice and plated onto $\mathrm{MH}$ agar plates. Following $18 \mathrm{~h}$ of incubation at $37^{\circ} \mathrm{C}$ CFUs were counted.

\section{Statistical analysis}

All values are expressed as the means \pm SEM. Difference between mean values of normally distributed data were analyzed using one-way ANOVA (Dunnett's t-test) and two-tailed Student's $t$-test. The results are considered statistically significant at $p<0.05$ or $p<0.01$.

\section{Results}

\section{The protective effect of the gut microbiota on $S$. aureus-induced mastitis}

To detect the involvement of the gut microbiota in mastitis, the mice were exposed to $S$. aureus $\left(1 \times 10^{5}, 1 \times 10^{6}\right.$, and $\left.1 \times 10^{7} \mathrm{CFU}\right)$ by infusion into the mammary gland. The results showed that gut microbiota-dysbiosis mice exhibited mammary gland histomorpholigic changes and increased infiltration of neutrophils (Fig. 1a and Supplementary Fig. S1); increased populations of neutrophils, macrophages, T cells, NK cells and reduced ratio of CD4+/CD8+ cells (see Supplementary Fig. S2A-D); increased TNF- $\alpha$, IL-1 $\beta$, IL-6 (Fig. 1b-d), and MPO levels (Fig. 1e); bloodmilk barrier permeability (as confirmed by reduced levels of Occludin, Claudin-3, and ZO-1 in mammary glands (Fig. 1f) and increases distribution of FITC-albumin in the mammary gland alveolar lumen (see Supplementary Fig. S3A)); and intestinal barrier disruption (as confirmed by reduced levels of Occludin, Claudin-3, and ZO-1 in intestinal tissues, see Supplementary Fig. S3B) compared with those of untreated mice. FMT to gut microbiotadysbiosis mice reversed these changes (Fig. 1a-f and Supplementary Figs. S1-S3). Furthermore, after infection with a concentration gradient of $S$. aureus, the gut microbiotadysbiosis mice exhibited more severe pathologic changes (Fig. 1a and Supplementary Fig. S1); increased populations of neutrophils, macrophages, T cells, and NK cells and reduced ratios of CD4+/CD8 + cells (see Supplementary Fig. S2); increased TNF- $\alpha$, IL-1 $\beta$, and IL-6 levels (Fig. 1b-d); and elevated MPO activity (Fig. 1e) compared with those of the untreated mice infected with $S$. aureus. However, after infection with a concentration gradient of $S$. aureus in mice subjected to FMT mice, these changes were significantly alleviated (Fig. 1a-e and Supplementary Figs. S1 and S2). Finally, we investigated whether increasing blood-milk barrier permeability could help keep the bacteria from leaving the mammary tissues and entering systemic circulation. Our results showed gut microbiotadysbiosis mice had increased bacterial loads in their blood $24 \mathrm{~h}$ after $S$. aureus exposure compared with those in the blood of untreated mice. However, FMT to gut microbiotadysbiosis mice resulted in lower bacterial loads than those in gut microbiota-dysbiosis mice (see Supplementary Fig. S3C).

\section{Characterization of the gut microbiota in mice after Abx (cocktail of antibiotics) treatment and FMT}

To evaluate the species richness and diversity of the gut bacterial community, we analyzed the observed species and, Shannon index from each sample. The results showed that the bacterial richness and diversity of the gut microbiota in the Abx group were significantly lower than those in any other group (see Supplementary Fig. S4A, B). In addition, Nonmetric multidimensional scaling (NMDS) ordination performed on the Bray-Curtis dissimilarity showed that the bacterial community profiles of feces from Abx-treated mice were separated from those of samples in the untreated group, while the bacterial community in the FMT groups was close to that in the untreated groups (see supplementary Fig. S4C). These results suggested that Abx treatment results in gut microbiota dysbiosis and that FMT reversed the bacterial community construction induced by Abx. Furthermore, detection of the bacterial phyla showed that the relative 


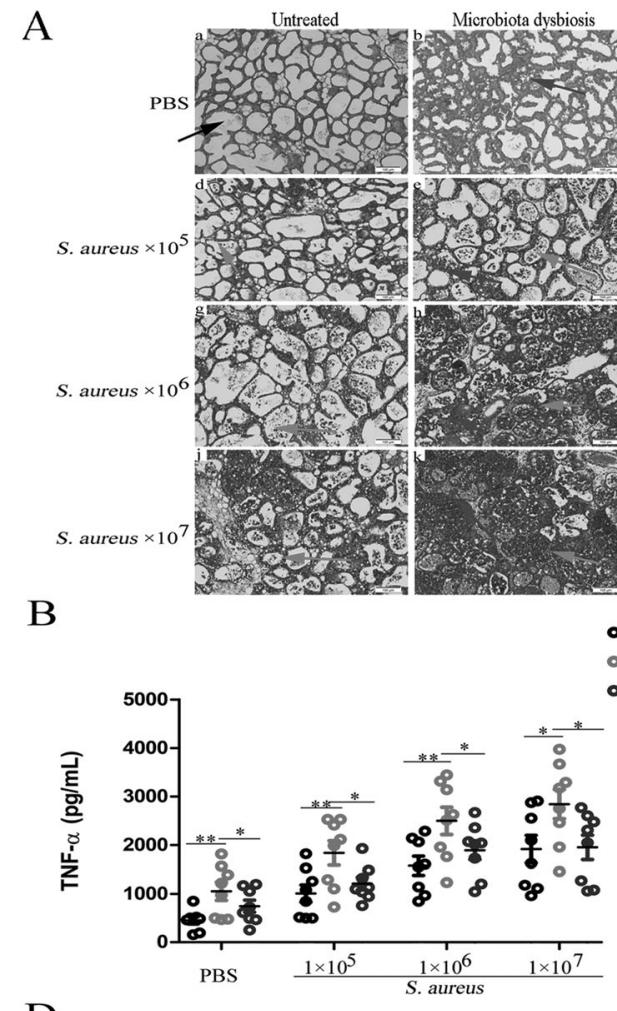

$\mathrm{D}$

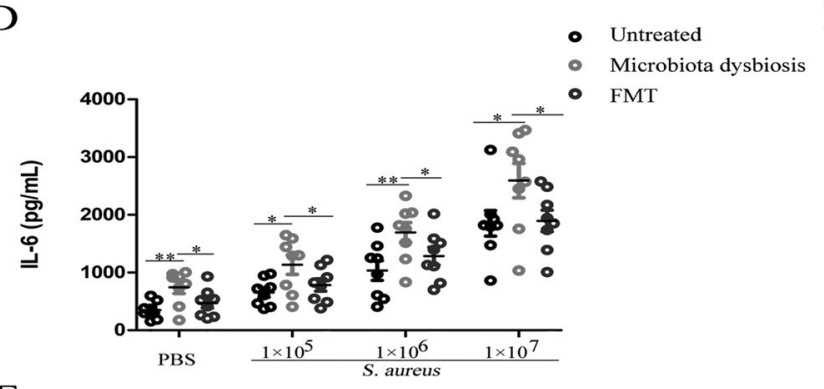

$\mathrm{F}$
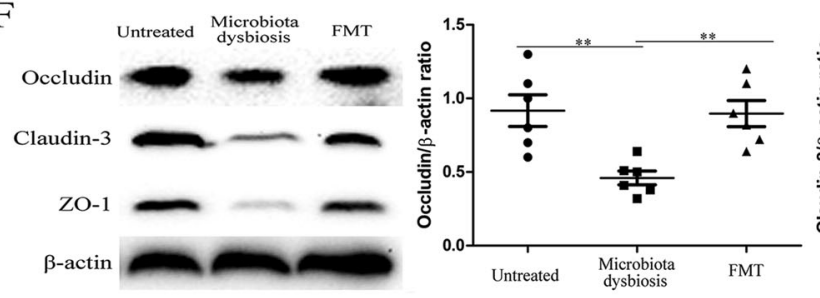

Fig. 1 The protective effect of gut microbiota on S. aureus-induced mastitis. Mammary gland tissues were harvested from untreated mice, gut microbiota-dysbiosis mice and FMT to gut microbiota-dysbiosis mice $24 \mathrm{~h}$ after infection with $S$. aureus $\left(1 \times 10^{5}, 1 \times 10^{6}\right.$, and $1 \times 10^{7}$ CFU). a Mammary gland tissue sections were stained with $\mathrm{H} \& \mathrm{E}$ $(100 \times)$. The black arrow was the normal tissues. The red arrow was the infiltration of inflammatory cells. The blue arrow was the hyperplastic

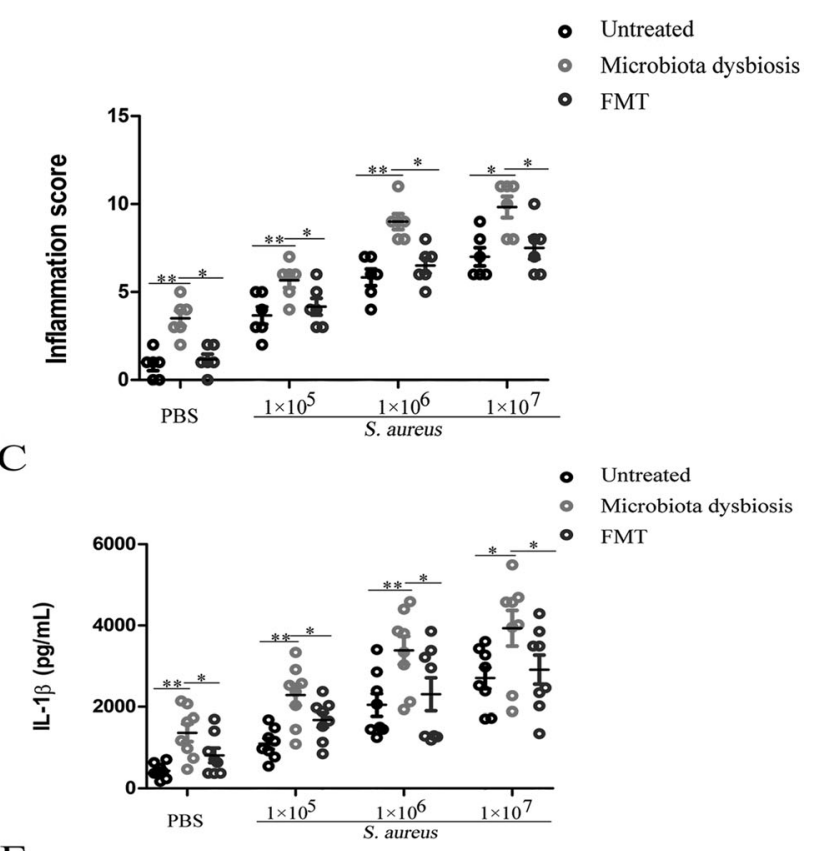

$\mathrm{E}$
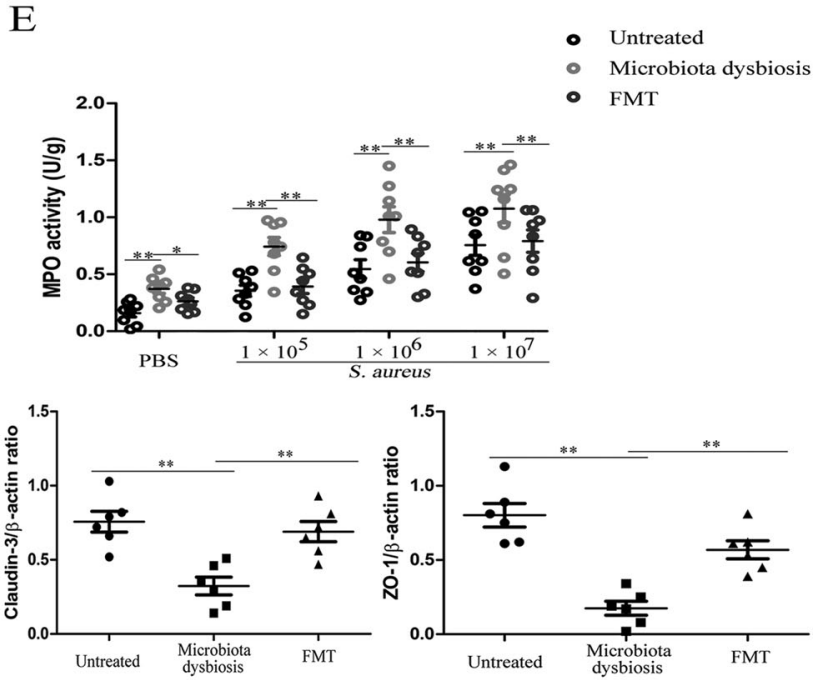

of alveolar wall. Levels of pro-inflammatory cytokines, including TNF- $\alpha(\mathbf{b})$, IL-1 $\beta$ (c), and IL-6 (d), and MPO activity (e) in mammary glands. f Levels of the tight junction proteins Occludin, Claudin-3, and ZO-1 in mammary glands. The results are presented as the mean \pm SEM of three independent experiments $(n=6-8) ; * p<0.05$ is significantly different from each group.

showed that the relative abundance of Enterobacter was significantly increased, while that of Lactobacillus and Bacteroides was decreased, and Prevotellaceae_UCG-003 abundance was not significantly different in the Abx treatment group compared with that in control group. However, this effect was markedly reversed by FMT (Fig. 2c, d). abundance of Proteobacteria was significantly increased, while the relative abundances of Firmicutes and Bacteroidetes were markedly reduced in the feces microbiota from Abx-treated mice compared with those in the feces microbiota of the untreated mice (Fig. 2a, b). At the genus level, the four dominant bacterial genera in the fecal microbiota 

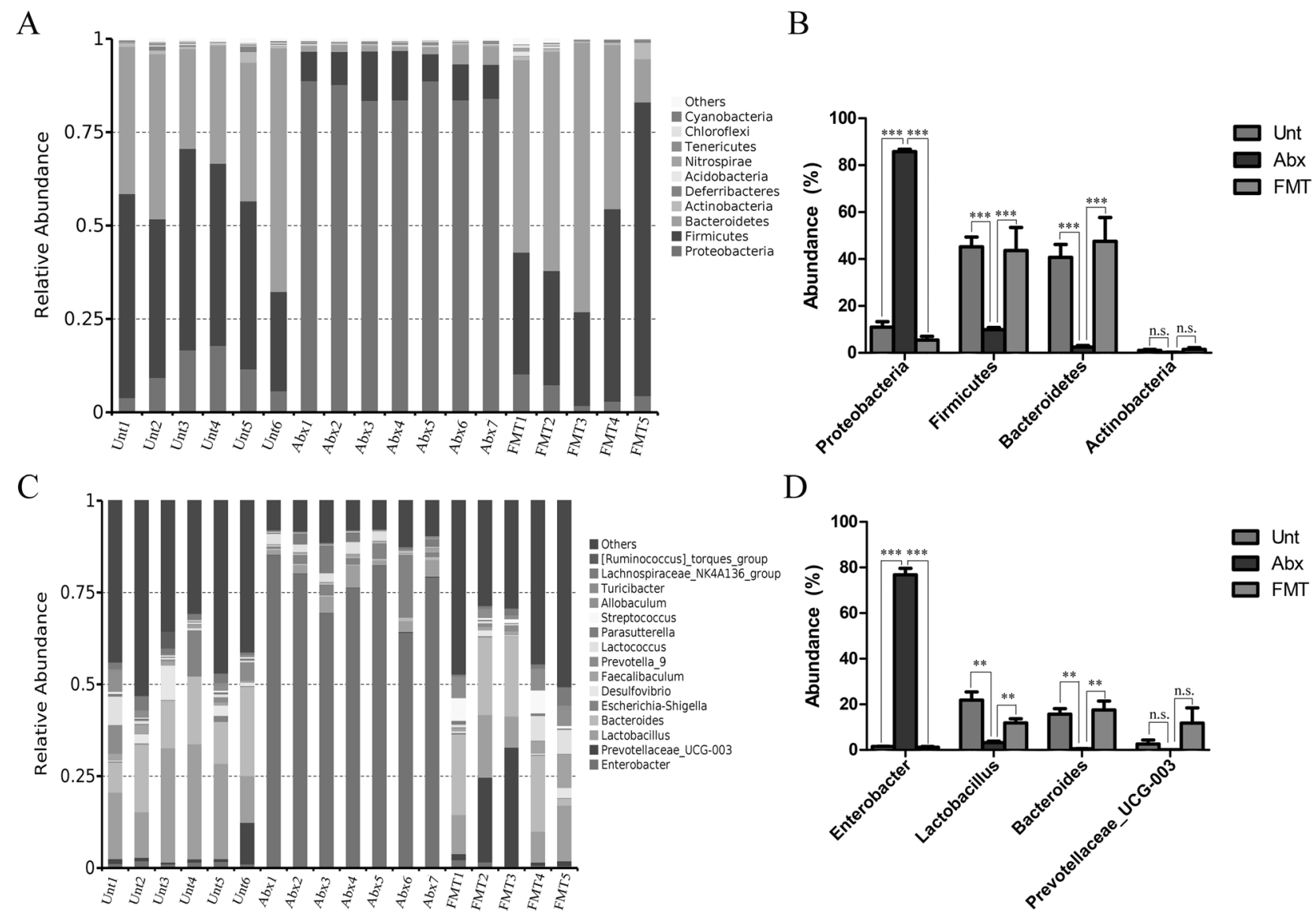

D

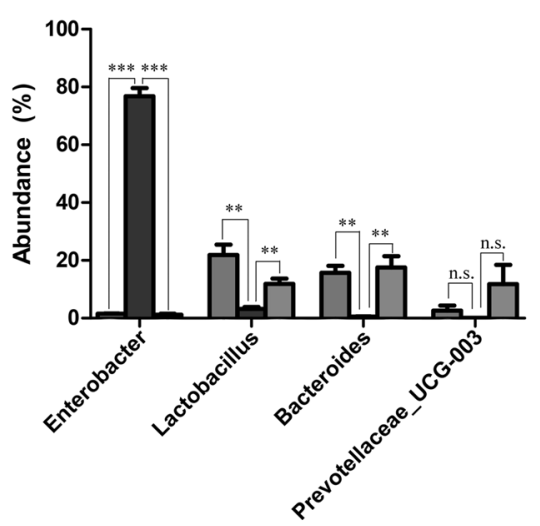

Fig. 2 Characterization of the gut microbiota in mice after Abx treatment and FMT. The feces microbiota from untreated, Abxtreated, and FMT to Abx-treated mice were detected by using the V4 region of the bacterial $16 \mathrm{~S}$ ribosomal RNA (rRNA) gene amplified PCR. a Relative abundance of the top 10 phyla in the feces. b Relative abundance of the four major phyla in the feces. c Relative abundance of the top 15 genera in the feces. d Relative abundance of the four major genera in the feces. The results are presented as the mean \pm SEM of three independent experiments $(n=5-7) ; * p<0.05$ is significantly different from each group.
Table 1 The concentrations of short-chain fatty acid in fecal.

\begin{tabular}{llllll}
\hline SCFAs & Unt $(\mu \mathrm{g} / \mathrm{g})$ & Abx $(\mu \mathrm{g} / \mathrm{g})$ & FMT $(\mu \mathrm{g} / \mathrm{g})$ & \multicolumn{2}{l}{$p$ value } \\
\cline { 5 - 6 } & & & Unt vs Abx & Abx vs FMT \\
\hline Acetate & $1280.74 \pm 363.72$ & $167.93 \pm 25.12$ & $1195.66 \pm 184.95$ & $* * *$ & $* * *$ \\
Propionate & $992.75 \pm 194.20$ & $9.77 \pm 2.51$ & $900.77 \pm 94.29$ & $* * *$ & $* * *$ \\
Isobutyrate & $25.46 \pm 3.99$ & NA & $18.55 \pm 9.50$ & $* * *$ & $* * *$ \\
Butyrate & $880.86 \pm 10.94$ & NA & $418.53 \pm 45.07$ & $* * *$ & $* * *$ \\
Isovalerate & $9.28 \pm 0.51$ & NA & $2.29 \pm 0.45$ & $* * *$ & $* * *$ \\
Valerate & $65.51 \pm 5.90$ & NA & $43.74 \pm 3.39$ & $* * *$ & $* * *$ \\
\hline
\end{tabular}

NA indicated undetectable.

${ }^{*} p$ value $<0.05$.

\section{Short-chain fatty acids contents are reduced in gut microbiota-dysbiosis mice}

To determine whether the protective effect of the gut microbiota on mastitis was associated with SCFAs, we assessed
SCFAs expression in mice. As shown in Table 1, acetate, propionate, and butyrate contents were significantly reduced in gut microbiota-dysbiosis mice compared with those in untreated mice. FMT to gut microbiota-dysbiosis mice restored the concentration of acetate, propionate, and butyrate. 


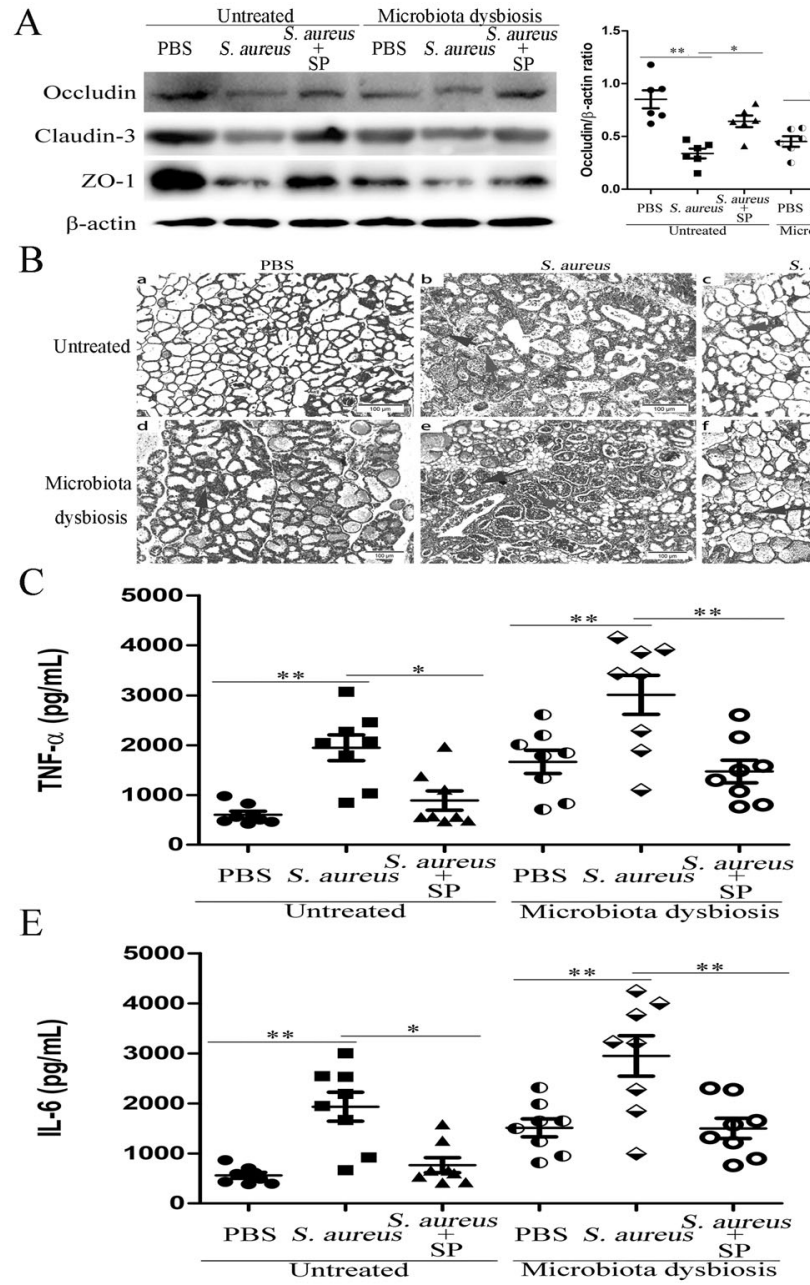

Fig. 3 Sodium propionate (SP) protects against S. aureus-induced mastitis by regulating blood-milk barrier permeability. Mice were administered an intraperitoneal injection of sodium propionate (SP, $100 \mathrm{mg} / \mathrm{kg}) 1 \mathrm{~h}$ before infection with $\mathrm{S}$. aureus $\left(1 \times 10^{7} \mathrm{CFU}\right)$. Twentyfour hours later, mammary gland tissues were collected and used for testing. a Expression of tight junction proteins, including Occludin, Claudin-3, and ZO-1, was tested $24 \mathrm{~h}$ after infection in untreated and gut microbiota-dysbiosis mice. b Mammary gland tissue sections of untreated and gut microbiota-dysbiosis mice were stained with $\mathrm{H} \& \mathrm{E}$

\section{Effects of SCFAs on S. aureus-induced mastitis}

The effects of SCFAs (acetate, propionate, and butyrate) on $S$. aureus-induced mastitis were detected. Our results showed that pretreatment with sodium propionate (SP) or sodium butyrate (SB) could reduce blood-milk barrier permeability (Fig. 3a and Supplementary Fig. S5A, or Fig. 4a and Supplementary S6A), mammary gland damage and inflammatory cells infiltration (Fig. 3b and Supplementary Fig. S5B or Fig. 4b and Supplementary S6B), production of pro-inflammatory cytokines (Fig. 3c-e or Fig. $4 \mathrm{c}-\mathrm{e}$ ), and MPO activity (Fig. $3 \mathrm{f}$ or $4 \mathrm{f}$ ) in the mammary gland in both untreated and gut microbiota-dysbiosis
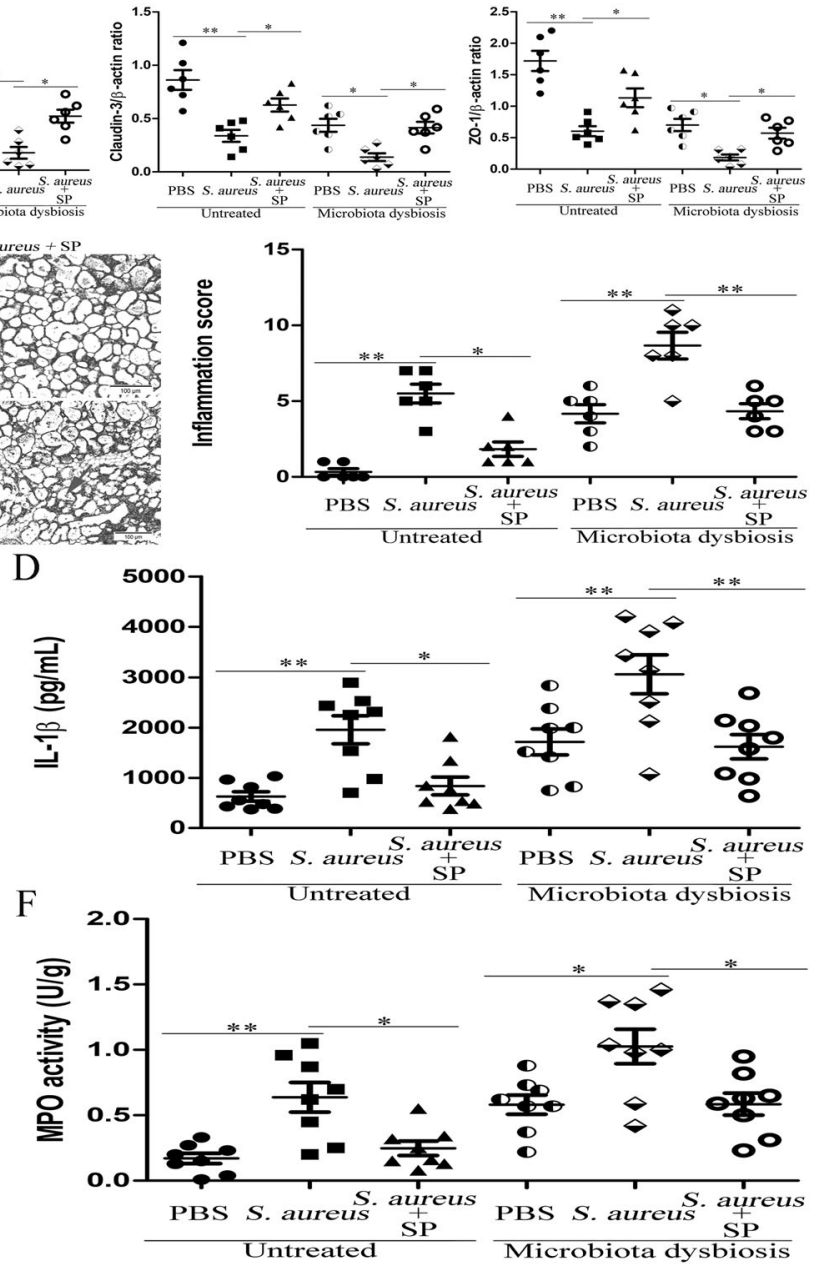

$(100 x)$. The black arrow was the normal tissues. The red arrow was the infiltration of inflammatory cells. The blue arrow was the hyperplastic of alveolar wall. Levels of pro-inflammatory cytokines, including TNF- $\alpha(\mathbf{c})$, IL-1 $\beta$ (d), and IL-6 (e), as well as MPO activity (f) in mammary glands was assessed $24 \mathrm{~h}$ after infection in untreated and gut microbiota-dysbiosis mice. The results are presented as the mean \pm SEM of three independent experiments $(n=6-8) ; * p<0.05$ is significantly different from each group.

mice after infection with $S$. aureus. Meanwhile, we found that the levels of propionate and butyrate were increased in both blood and mammary gland tissues after treatment with SP or SB (see supplementary Table S2). In addition, we also found that SP and SB did not affect $S$. aureus growth (see Supplementary Fig. S5C and Supplementary Fig. S6C). These data suggested that SP and SB could protect against $S$. aureus-induced mastitis through reducing the inflammatory response and regulating blood-milk barrier permeability. Furthermore, we found that treatment with sodium acetate (SA) had no protective effect on mastitis induced by $S$. aureus (Fig. 5a-e and Supplementary Fig. S7). 

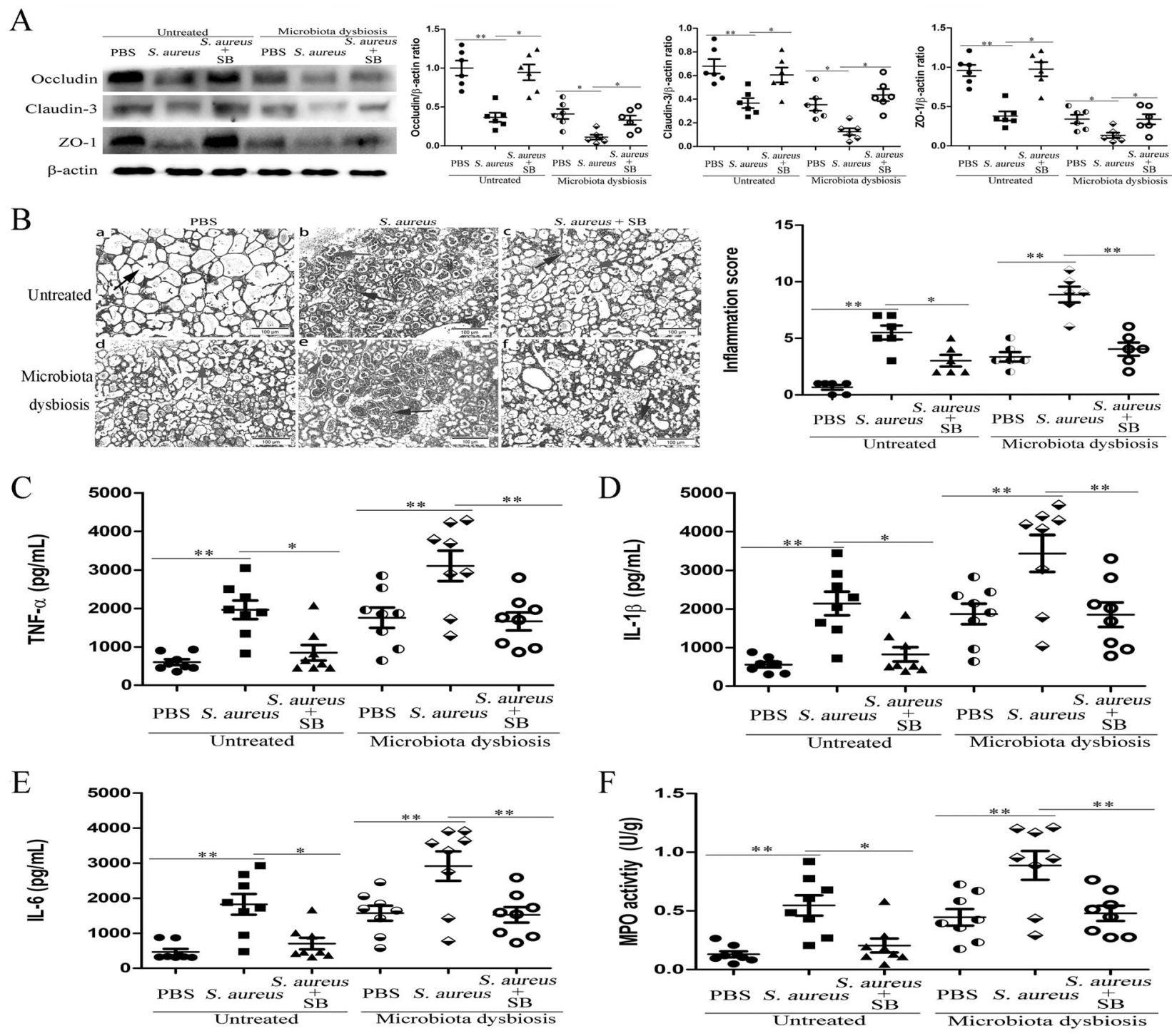

Fig. 4 Sodium butyrate (SB) protects against S. aureus-induced mastitis by regulating blood-milk barrier permeability. Mice were administered an intraperitoneal injection of sodium butyrate (SB, $100 \mathrm{mg} / \mathrm{kg}) 1 \mathrm{~h}$ before infection with S. aureus $\left(1 \times 10^{7} \mathrm{CFU}\right)$. Twentyfour hours later, the mammary gland tissues were collected and assessed. a Levels of the tight junction proteins Occludin, Claudin-3, and Occludin in mammary glands were assessed $24 \mathrm{~h}$ after infection in untreated mice and gut microbiota-dysbiosis mice. b Mammary gland tissue sections of untreated mice and gut microbiota-dysbiosis mice

\section{C. tyrobutyricum protects against S. aureus-induced mastitis through the production of butyrate}

The effects of $C$. tyrobutyricum, a bacterium that can produce butyrate, on $S$. aureus-induced mastitis were detected in this study. Our results showed that $C$. tyrobutyricum significantly reduced $S$. aureus-induced blood-milk barrier permeability (Fig. 6a and Supplementary Fig. S8A), mammary gland pathologic damage (Fig. $6 \mathrm{~b}$ and Supplementary Fig. S8B), pro-inflammatory cytokine (TNF- $\alpha$, IL- $1 \beta$, and

were stained with $\mathrm{H} \& \mathrm{E}(100 \times)$. The black arrow was the normal tissues. The red arrow was the infiltration of inflammatory cells. The blue arrow was the hyperplastic of alveolar wall. Levels of proinflammatory cytokines, including TNF- $\alpha$ (c), IL-1 $\beta$ (d), and IL-6 (e), as well as MPO activity (f) in mammary glands were assessed in mammary glands $24 \mathrm{~h}$ after infection in untreated mice and gut microbiota-dysbiosis mice. The results are presented as the mean \pm SEM of three independent experiments $(n=6-8) ; * p<0.05$ is significantly different from each group.

IL-6) production (Fig. 6c-e), and MPO activity (Fig. 6f). The above results suggested that $C$. tyrobutyricum protected against $S$. aureus-induced mastitis through reducing the inflammatory response and regulating blood-milk barrier permeability. Subsequently, to detect the protective effects of $C$. tyrobutyricum on $S$. aureus-induced mastitis through the potential components of bacteria or the production of butyrate, we assessed the effects of heat-inactivated $C$. tyrobutyricum and $C$. tyrobutyricum supernatant on $S$. aureus-induced mastitis. The results showed that $C$. 

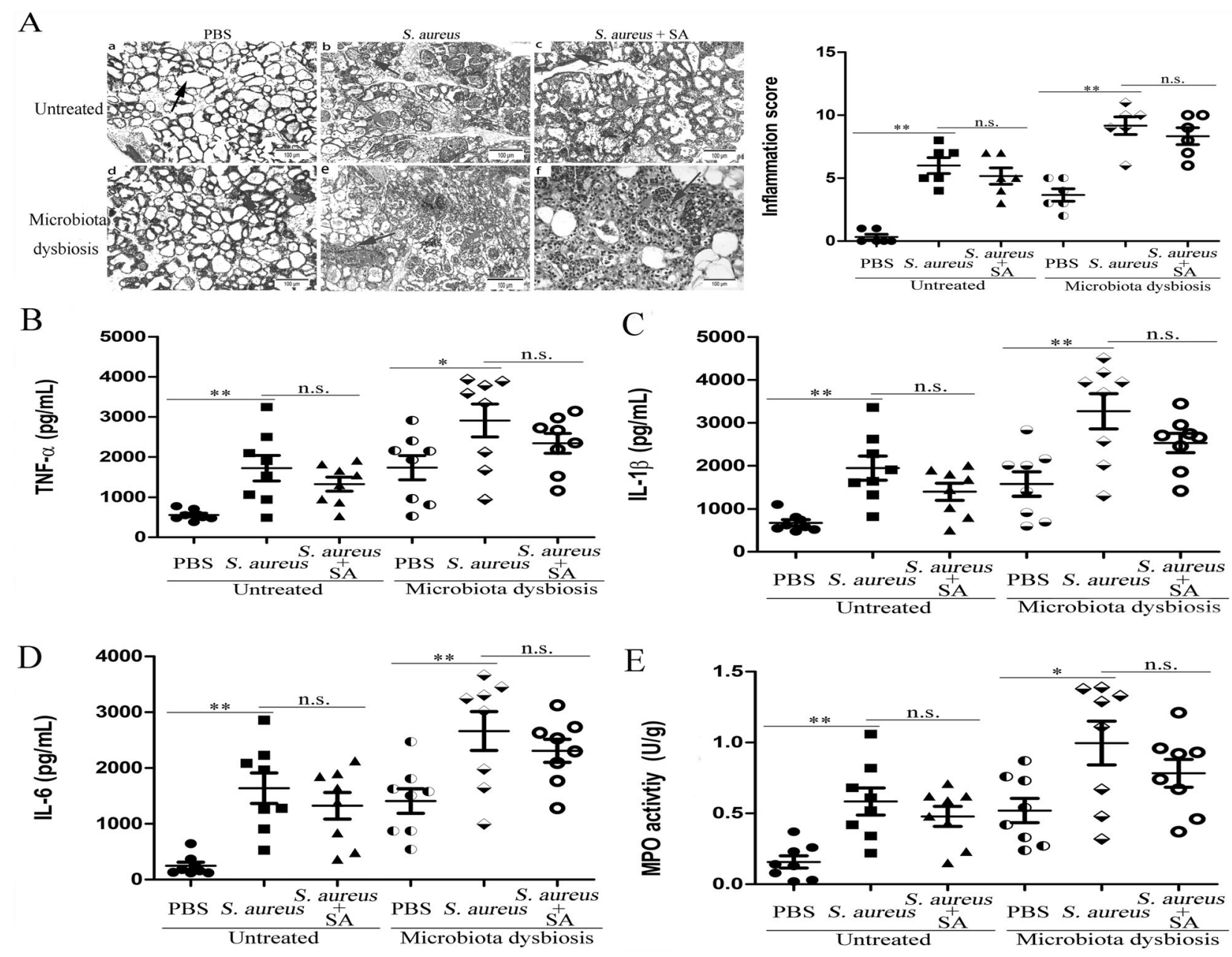

Fig. 5 Sodium acetate (SA) has no protective effect on mastitis induced by $\mathbf{S}$. aureus. Mice were administered an intraperitoneal injection of sodium acetate (SA, $100 \mathrm{mg} / \mathrm{kg}$ ) $1 \mathrm{~h}$ before infection with S. aureus $\left(1 \times 10^{7} \mathrm{CFU}\right)$. Twenty-four hours later, mammary gland tissues were collected and used for testing. a Mammary gland tissue sections of untreated and gut microbiota-dysbiosis mice were stained with $H \&$ E (100x). The black arrow was the normal tissues. The red

tyrobutyricum supernatant but not heat-inactivated $C$. tyrobutyricum alleviated mammary gland pathological damage and inflammatory cells infiltration (Fig. 7a and Supplementary Fig. S9) and the production of the proinflammatory cytokines TNF- $\alpha$, IL-1 $\beta$, and IL-6 induced by $S$. aureus (Fig. 7b-d). Furthermore, we measured the levels of butyrate in C. tyrobutyricum supernatant and the levels of propionate and butyrate in the blood and mammary glands of $C$. tyrobutyricum group mice. The levels of butyrate in $C$. tyrobutyricum supernatant was showed in Table S2 and supplementation of $C$. tyrobutyricum visibly increased the levels of butyrate and propionate in the blood and the levels of butyrate in the mammary gland tissues (Table S3). These results suggested that the protective effects of $C$. tyrobutyricum on $S$. aureus-induced mastitis may be due to butyrate production.

arrow was the infiltration of inflammatory cells. The blue arrow was the hyperplastic of alveolar wall. Levels of pro-inflammatory cytokines, including TNF- $\alpha(\mathbf{b})$, IL-1 $\beta$ (c), and IL-6 (d), as well as MPO activity (e) in mammary gland tissues was tested $24 \mathrm{~h}$ after infection in untreated and gut microbiota-dysbiosis mice. The results are presented as the mean \pm SEM of three independent experiments $(n=6-8)$; ${ }^{*} p<$ 0.05 is significantly different from each group.

\section{Discussion}

The results of this study demonstrated that gut microbiota dysbiosis is an important factor in the host that contributes to the severity of mastitis. The data support the idea that gut microbiota dysbiosis results in changes in mammary gland histomorphology and increases the inflammation score, as well as the levels of immune cells. In addition, mammary gland involution is a highly complex multistep process and is a highly dynamic and time-dependent process that results in defferences in immune cell recruitment. Studies have suggested that the number of neutrophils in the mammary gland is slightly increased $48 \mathrm{~h}$ after weaning, followed by an accumulation of macrophages [27, 28]. These results suggested that the disorder of the gut microbiota induced by Abx seems to impact the timeline of mammary gland 

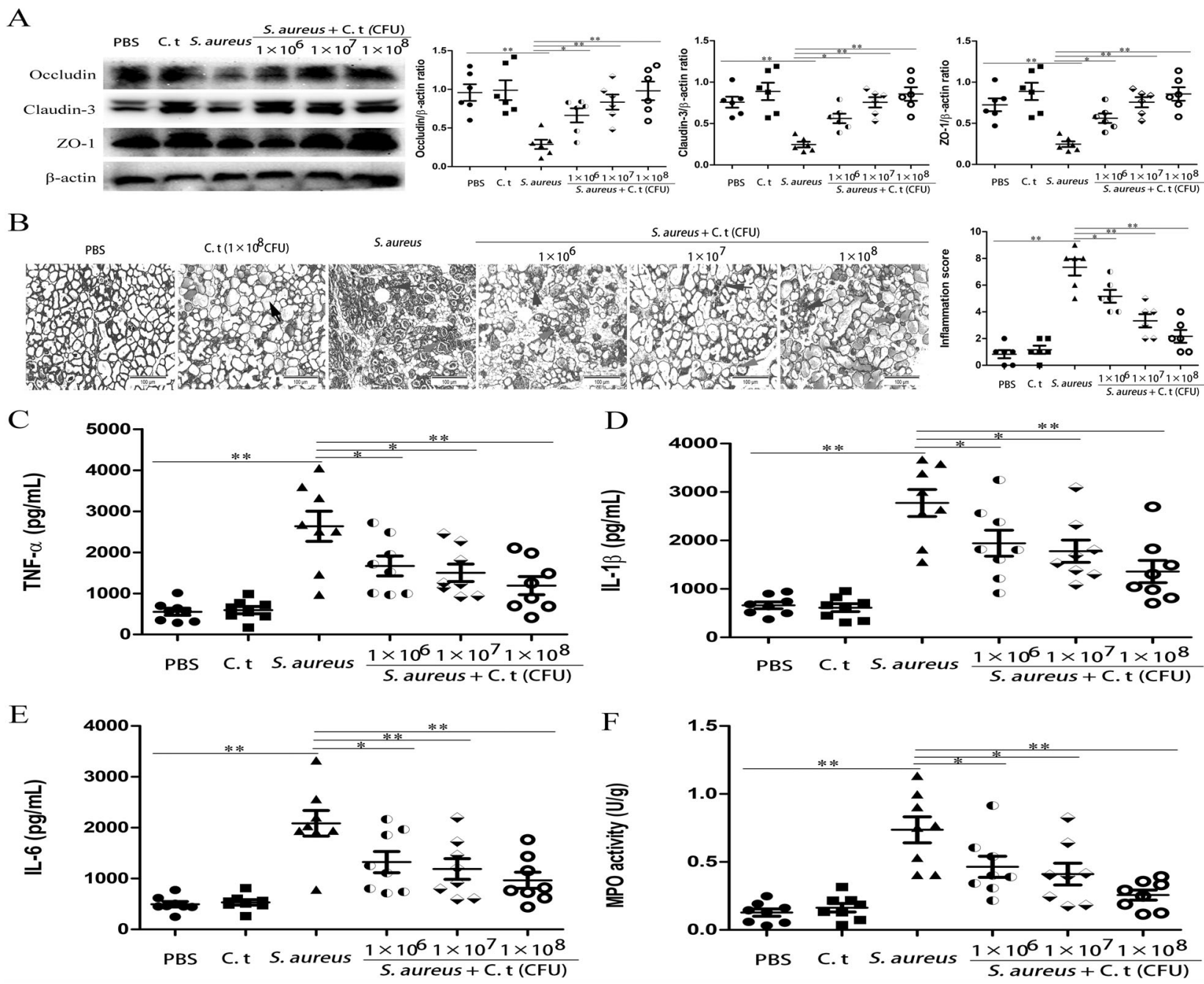

Fig. 6 C. tyrobutyricum protects against S. aureus-induced mastitis by regulating blood-milk barrier. Mice were administered an oral gavage of $C$. tyrobutyricum $\left(1 \times 10^{6}, 1 \times 10^{7}\right.$, and $1 \times 10^{8} / 200 \mu \mathrm{L}$ PBS) for 7 consecutive days and then were infected with $S$. aureus $\left(1 \times 10^{7} \mathrm{CFU}\right)$. Twenty-four hours later, mammary gland tissues were collected and used for testing. a Expression of tight junction proteins, including Occludin, Claudin-3, and ZO-1 in mammary glands. b Mammary gland tissue sections of mice were stained with

H \& E (100x). The black arrow was the normal tissues. The red arrow was the infiltration of inflammatory cells. The blue arrow was the hyperplastic of alveolar wall. Levels of pro-inflammatory cytokines, including TNF- $\alpha(\mathbf{c})$, IL-1 $\beta$ (d), and IL-6 (e), as well as MPO activity (f) in mammary glands. The results are presented as the mean \pm SEM of three independent experiments $(n=6-8) ;{ }^{*} p<0.05$ is significantly different from each group.

involution. This finding also showed that our experimental model has certain limitations that could impact this conclusion. Furthermore, after infection with $S$. aureus, inflammatory injury was significantly increased in gut microbiota-dysbiosis mice compared with that in untreated mice. However, FMT to gut microbiota-dysbiosis mice significantly reversed these changes.

The blood-milk barrier is a specific structure that plays an important role in protecting mammary gland function in mammals. Increased permeability of the blood-milk barrier leads to a massive recruitment of somatic cells, in particular PMNs. Increased PMNs in the mammary gland could exacerbate mammary gland inflammation [25, 29-31]. Some evidence has suggested that the gut microbiota plays

an important role in the development of the blood-brain barrier (BBB) [20, 32, 33]. Therefore, we hypothesized that the gut microbiota may affect the development of the bloodmilk barrier. Pre-established dysbiosis using Abx significantly reduced the tight junction protein content, including that of Occludin, Claudin-3, and ZO-1, of the blood-milk barrier. However, FMT to gut microbiotadysbiotic mice increased Occludin, Claudin-3 and ZO-1 levels. FITC-albumin is another important factor used to evaluate blood-milk barrier permeability [25]. Our results demonstrated that FITC-albumin was distributed in the alveolar lumen in gut microbiota-dysbiosis mice. However, fluorescent reactions were not present in the alveolar lumen of untreated mice. FMT to gut microbiota-dysbiotic mice 
A
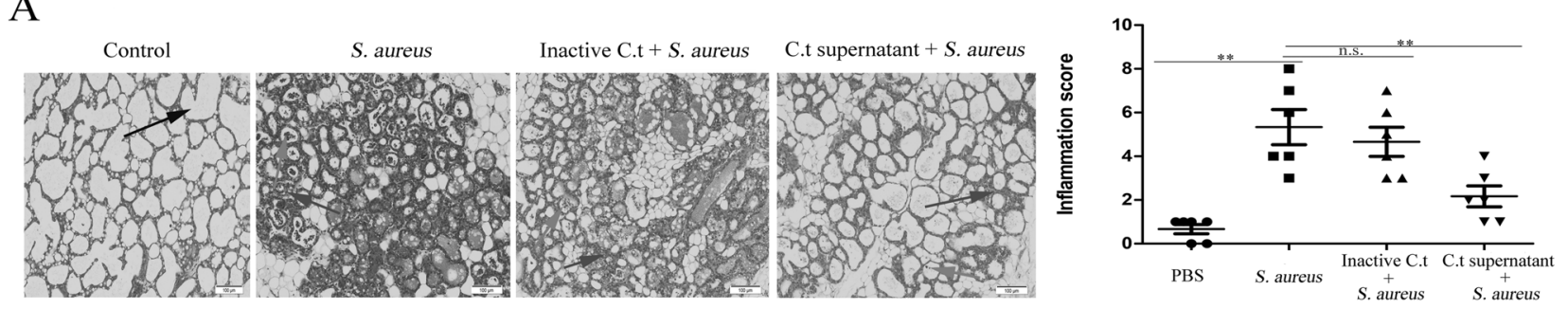

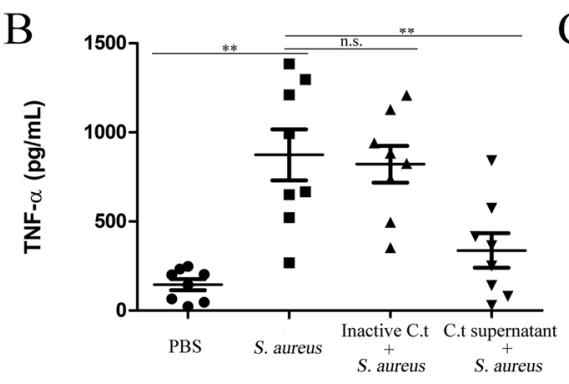

$\mathrm{C}$

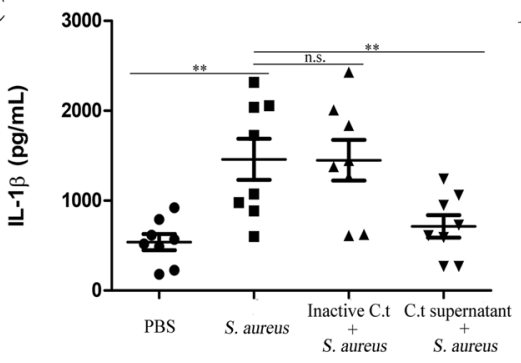

$\mathrm{D}$

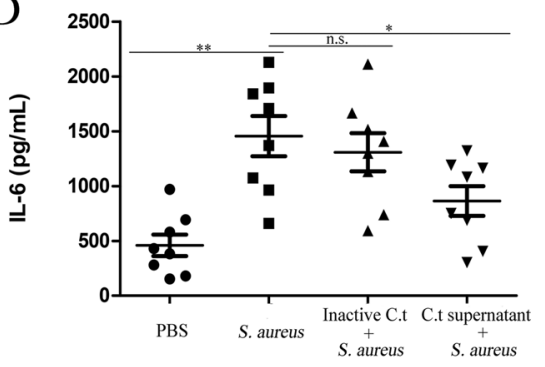

Fig. 7 C. tyrobutyricum protects against $S$. aureus-induced mastitis through the production of butyrate. Mice were administered an oral gavage of heat-inacitvated $C$. tyrobutyricum $\left(1 \times 10^{8} / 200 \mu \mathrm{L}\right.$ PBS $)$ or C. tyrobutyricum supernatant for 7 consecutive days and then were infected with $S$. aureus $\left(1 \times 10^{7} \mathrm{CFU}\right)$. Twenty-four hours later, mammary gland tissues were collected and used for testing. (A) Mammary gland tissue sections of mice were stained with $\mathrm{H} \& \mathrm{E}$
$(100 \times)$. The black arrow was the normal tissues. The red arrow was the infiltration of inflammatory cells. The blue arrow was the hyperplastic of alveolar wall. Levels of pro-inflammatory cytokines, including TNF- $\alpha(B)$, IL-1 $\beta(C)$, and IL-6 (D), in mammary glands. The results are presented as the mean \pm SEM of three independent experiments $(n=6-8) ; * p<0.05$ is significantly different from each group.

pneumonia, colorectal cancer, sepsis [35-37], and mastitis $[38,39]$. Thus, the increased abundance of Enterobacter in the gut microbiota of Abx-treated mice maybe associated with the inflammatory response of the mammary gland.

In addition, the Firmicutes phylum and Bacteroides are bacterial that produce SCFAs. Increasing evidence suggests that the gut microbiota represents a vital factor to host health, at least in part through the release of SCFAs, which are produced by the gut microbiota after the fermentation of partially and non-digestible polysaccharides [9, 40]. The evidence suggested that propionate plays an important role in host susceptibility to bacterial and fungal infections [41]. Recently, it has been demonstrated that propionate protects against LPS-induced mastitis through regulating the bloodmilk barrier [42]. The evidence also demonstrated that SCFAs, especially butyrate, play a central role in modulating the inflammatory response $[43,44]$. On the other hand, butyrate regulates intestinal cell growth and differentiation and induces angiogenesis in the small intestine [45]. Above all, butyrate is an most important nutrient that induces the expression of tight junction proteins [46]. These findings suggest that SCFAs participate in the development of the blood-milk barrier. Our data indicate that acetate, propionate, and butyrate levels were significantly decreased and that SCFAs expression was restored after FMT to gut microbiota-dysbiosis mice. Furthermore, pretreated mice with sodium propionate (SP), sodium butyrate (SB), rather than sodium acetate (SA) in the context of $S$. aureus- is $[34,35]$. Enterobacter is an important pathogen contributes to the development of many diseases, including 
induced mastitis alleviated inflammatory biomarkers through enhancing blood-milk barrier function both in untreated mice and gut microbiota-dysbiosis mice. To further research the mechanisms by which SP and SB inhibit pathology, we assessed the levels of propionate and butyrate in the blood and mammary glands and the effect of SP and SB on $S$. aureus growth. Our data demonstrated that both propionate and butyrate levels were increased in the blood and mammary glands after SP or SB treatment. In addition, neither SP nor SB affected $S$. aureus growth. These data suggest that the protective effect of SP and SB against $S$. aureus-induced mastitis occurs via inhibition of the proinflammatory immune response maintenance of blood-milk barrier permeability.

Finally, we treated animals with butyrate-producing bacteria (C. tyrobutyricum) and observed a protective role against $S$. aureus-induced mastitis. $C$. tyrobutyricum resides in the gut and has a protective role in defending against pathogenic bacteria by regulating of gut microbiota metabolites, such as butyrate [47, 48]. Recent evidence indicates that colonization of germ-free mice with $C$. tyrobutyricum restores the function of TJs in the BBB or BTB $[19,20]$. In our study, we also found that $C$. tyrobutyricum reduced blood-milk barrier permeability and alleviated inflammation of the mammary gland induced by $S$. aureus. It is suggested that metabolites from the gut microbiota are important for controlling inflammation in distant organs. Additional supporting evidence showed that treatment with $C$. tyrobutyricum improved kidney function by modulating the inflammatory process during ischemia-reperfusion-induced acute kidney injury [49]. To clarify the protective mechanism of $C$. tyrobutyricum on mastitis, we orally administered of heat-inactivated $C$. tyrobutyricum and $C$. tyrobutyricum supernatants and then induced mastitis by infecting $S$. aureus. Interestingly, $C$. tyrobutyricum supernatant bot not heat-inactivated $C$. tyrobutyricum inhibited $S$. aureus-induced mastitis. In addition, the levels of butyrate in both the blood and mammary gland were also significantly increased in mice that received live $C$. tyrobutyricum. Furthermore, the results showed SP administration not only elevated the concentration of propionate but also increased the concentration of butyrate in the mammary gland. This result may be associated with the addition of propionate promoting the synthesis of butyrate. To support our hypothesis, the research proved the existence of propionate fermentation, and butyrate is synthesized by an elongation of the carbon chain of propionate [50]. Furthermore, the concentrations of butyrate and propionate were higher in mammary gland tissues than in circulating plasma after administration of SB and SP, which may be due to a large amount of butyrate and propionate entering the mammary gland along with the blood and accumulating in the mammary gland during lactation. It is suggested that the protective effects of $C$. tyrobutyricum against mastitis are partly due to the anti-inflammatory property of its metabolite butyrate and the idea that butyrate-producing bacteria potentially act as a tool for the management or prevention of inflammatory processes.

In conclusion, our findings demonstrate that the gut microbiota improves organ function after damage via regulation of the inflammatory process and modulation of blood-milk barrier permeability in a model of the mastitis induced by $S$. aureus. These effects may be partly attributed to the regulation of Enterobacter and SCFAs production. Probiotics or drugs that regulate the gut-mammary gland axis through regulating gut microbiota balance may represent a new promising direction for mastitis treatment (Fig. S10).

Acknowledgements This work was supported by a grant from the National Natural Science Foundation of China (Nos. 31772812, 31972749, 31472248, 30972225, 30771596) and China Postdoctoral Science Foundation funded project (2016M600233).

\section{Compliance with ethical standards}

Conflict of interest The authors declare that they have no conflict of interest.

Ethical statement The permit number (20170317) was assigned by the Institutional Animal Care and Use Committee (IACUC) of Jilin University for animal experiments approvals. The full proposal was reviewed by the IACUC ethics committee, which approved the animal care and use permit license. All experiments comply with the manual of the care and use of laboratory animals published by the US National Institutes of Health.

Publisher's note Springer Nature remains neutral with regard to jurisdictional claims in published maps and institutional affiliations.

\section{References}

1. Melchior MB, Vaarkamp H, Fink-Gremmels J. Biofilms: a role in recurrent mastitis infections? Vet J. 2006;171:398-407.

2. Castilho IG, Dantas STA, Langoni H, Araujo JP Jr, Fernandes A, $\mathrm{Jr}$, Alvarenga FCL, et al. Host-pathogen interactions in bovine mammary epithelial cells and HeLa cells by Staphylococcus aureus isolated from subclinical bovine mastitis. J Dairy Sci. 2017;100:6414-21.

3. Azara E, Longheu C, Sanna G, Tola S. Biofilm formation and virulence factor analysis of Staphylococcus aureus isolates collected from ovine mastitis. J Appl Microbiol. 2017;123:372-9.

4. Yue Y, Hymoller L, Jensen SK, Lauridsen C, Purup S. Effects of vitamin D and its metabolites on cell viability and Staphylococcus aureus invasion into bovine mammary epithelial cells. Vet Microbiol. 2017;203:245-51.

5. Smith GW, Lyman RL, Anderson KL. Efficacy of vaccination and antimicrobial treatment to eliminate chronic intramammary Staphylococcus aureus infections in dairy cattle. J Am Vet Med Assoc. 2006;228:422-5.

6. Ziesch M, Wente N, Zhang Y, Zaremba W, Engl S, Kromker V. Noninferiority trial investigating the efficacy of a nonantibiotic intramammary therapy in the treatment of mild-to-moderate 
clinical mastitis in dairy cows with longer lasting udder diseases. $\mathbf{J}$ Vet Pharm Ther. 2018;41:11-21.

7. Coyte KZ, Rakoff-Nahoum S. Understanding competition and cooperation within the mammalian gut microbiome. Curr Biol. 2019;29:R538-R544.

8. Janssen AWF, Houben T, Katiraei S, Dijk W, Boutens L, van der Bolt $\mathrm{N}$, et al. Modulation of the gut microbiota impacts nonalcoholic fatty liver disease: a potential role for bile acids. J Lipid Res. 2017;58:1399-416.

9. Ohira H, Tsutsui W, Fujioka Y. Are short chain fatty acids in gut microbiota defensive players for inflammation and atherosclerosis? J Atheroscler Thromb. 2017;24:660-72.

10. Vetizou M, Daillere R, Zitvogel L. Gut microbiota and efficacy of cancer therapies. Biol Aujourdhui. 2017;211:51-67.

11. Wen L, Duffy A. Factors influencing the gut microbiota, inflammation, and type 2 diabetes. J Nutr. 2017;147:1468S-1475S.

12. Yu LX, Schwabe RF. The gut microbiome and liver cancer: mechanisms and clinical translation. Nat Rev Gastroenterol Hepatol. 2017;14:527-39.

13. Zhao G, Vatanen T, Droit L, Park A, Kostic AD, Poon TW, et al. Intestinal virome changes precede autoimmunity in type I diabetessusceptible children. Proc Natl Acad Sci USA. 2017;114:E6166-E6175.

14. Ma C, Zhao J, Xi X, Ding J, Wang H, Zhang H, et al. Bovine mastitis may be associated with the deprivation of gut Lactobacillus. Benef Microbes. 2016;7:95-102.

15. Addis MF, Tanca A, Uzzau S, Oikonomou G, Bicalho RC, Moroni P. The bovine milk microbiota: insights and perspectives from -omics studies. Mol Biosyst. 2016;12:2359-72.

16. Ma C, Sun Z, Zeng BH, Huang S, Zhao J, Zhang Y, et al. Cow-tomouse fecal transplantations suggest intestinal microbiome as one cause of mastitis. Microbiome. 2018;6:200.

17. Guidry AJ, O'Brien CN, Douglass LW. A bovine mammary endothelial/epithelial cell culture model of the blood/milk barrier. Can J Vet Res. 1998;62:117-21.

18. Wall SK, Hernandez-Castellano LE, Ahmadpour A, Bruckmaier RM, Wellnitz O. Differential glucocorticoid-induced closure of the blood-milk barrier during lipopolysaccharide- and lipoteichoic acid-induced mastitis in dairy cows. J Dairy Sci. 2016;99:7544-53.

19. Al-Asmakh M, Stukenborg JB, Reda A, Anuar F, Strand ML, Hedin L, et al. The gut microbiota and developmental programming of the testis in mice. PLoS One. 2014;9:e103809.

20. Braniste V, Al-Asmakh M, Kowal C, Anuar F, Abbaspour A, Toth $\mathrm{M}$, et al. The gut microbiota influences blood-brain barrier permeability in mice. Sci Transl Med. 2014;6:263ra158.

21. Schuijt TJ, Lankelma JM, Scicluna BP, e Melo FD, Roelofs JJTH, de Boer JD, et al. The gut microbiota plays a protective role in the host defence against pneumococcal pneumonia. Gut. 2016;65:575-83.

22. Tian ZH, Liu J, Liao MY, Li WJ, Zou JQ, Han XX, et al. Beneficial effects of fecal microbiota transplantation on ulcerative colitis in mice. Dig Dis Sci. 2016;61:2262-71.

23. Fu YH, Zhou ES, Wei ZK, Liang DJ, Wang W, Wang TC, et al. Glycyrrhizin inhibits the inflammatory response in mouse mammary epithelial cells and a mouse mastitis model. Febs $\mathrm{J}$. 2014;281:2543-57.

24. Cani PD, Amar J, Iglesias MA, Knauf C, Neynhck A, Alessi MC, et al. Metabolic endotoxemia initiates obesity and insulin resistance. Ann Nutr Metab. 2007;51:79-79.

25. Kobayashi K, Oyama S, Numata A, Rahman MM, Kumura H. Lipopolysaccharide disrupts the milk-blood barrier by modulating claudins in mammary alveolar tight junctions. Plos One. 2013;8: e62187.

26. Nguyen DA, Parlow AF, Neville MC. Hormonal regulation of tight junction closure in the mouse mammary epithelium during the transition from pregnancy to lactation. J Endocrinol. 2001;170:347-56.
27. Stein T, Morris JS, Davies CR, Weber-Hall SJ, Duffy MA, Heath VJ, et al. Involution of the mouse mammary gland is associated with an immune cascade and an acute-phase response, involving LBP, CD14 and STAT3. Breast Cancer Res. 2004;6: R75-R91.

28. Stein T, Salomonis N, Gusterson BA. Mammary gland involution as a multi-step process. J Mammary Gland Biol. 2007;12:25-35.

29. Mehrzad J, Duchateau L, Burvenich C. Viability of milk neutrophils and severity of bovine coliform mastitis. J Dairy Sci. 2004;87:4150-62.

30. Wellnitz O, Wall SK, Saudenova M, Bruckmaier RM. Effect of intramammary administration of prednisolone on the blood-milk barrier during the immune response of the mammary gland to lipopolysaccharide. Am J Vet Res. 2014;75:595-601.

31. Wellnitz O, Zbinden C, Huang X, Bruckmaier RM. Short communication: Differential loss of bovine mammary epithelial barrier integrity in response to lipopolysaccharide and lipoteichoic acid. J Dairy Sci. 2016;99:4851-6.

32. Harrington M. For lack of gut microbes, the blood-brain barrier 'leaks'. Lab Anim (NY). 2015;44:6.

33. Michel L, Prat A. One more role for the gut: microbiota and blood brain barrier. Ann Transl Med. 2016;4:15.

34. Rosean CB, Bostic RR, Ferey JCM, Feng TY, Azar FN, Tung KS, et al. Preexisting commensal dysbiosis is a host-intrinsic regulator of tissue inflammation and tumor cell dissemination in hormone receptor-positive breast cancer. Cancer Res. 2019;79:3662-75.

35. Yuan L, Zhang SR, Li H, Yang F, Mushtaq N, Ullah S, et al. The influence of gut microbiota dysbiosis to the efficacy of 5Fluorouracil treatment on colorectal cancer. Biomed Pharmacother. 2018;108:184-93.

36. Chou DW, Wu SL, Lee CT. Intensive care unit-acquired complicated necrotizing pneumonia caused by Enterobacter cloacae: a case report. Intractable Rare Dis. 2018;7:283-6.

37. Crapser J, Ritzel R, Verma R, Venna VR, Liu F, Chauhan A, et al. Ischemic stroke induces gut permeability and enhances bacterial translocation leading to sepsis in aged mice. Aging (Albany NY). 2016;8:1049-63.

38. Gao J, Barkema HW, Zhang L, Liu G, Deng Z, Cai L, et al. Incidence of clinical mastitis and distribution of pathogens on large Chinese dairy farms. J Dairy Sci. 2017;100:4797-806.

39. Gutierrez-Chavez AJ, Martinez-Ortega EA, Valencia-Posadas M, Leon-Galvan MF, Fuente-Salcido N, Bideshi DK, et al. Potential use of Bacillus thuringiensis bacteriocins to control antibioticresistant bacteria associated with mastitis in dairy goats. Folia Microbiol. 2016;61:11-9.

40. Tan J, McKenzie C, Potamitis M, Thorburn AN, Mackay CR, Macia L. The role of short-chain fatty acids in health and disease. Adv Immunol. 2014;121:91-119.

41. Ciarlo E, Heinonen T, Herderschee J, Fenwick C, Mombelli M, Le Roy D, et al. Impact of the microbial derived short chain fatty acid propionate on host susceptibility to bacterial and fungal infections in vivo. Sci Rep. 2016;6:37944.

42. Wang J, Wei Z, Zhang X, Wang Y, Yang Z, Fu Y. Propionate protects against lipopolysaccharide-induced mastitis in mice by restoring blood-milk barrier disruption and suppressing inflammatory response. Front Immunol. 2017;8:1108.

43. Lasitschka F, Giese T, Paparella M, Kurzhals SR, Wabnitz G, Jacob K, et al. Human monocytes downregulate innate response receptors following exposure to the microbial metabolite nbutyrate. Immun Inflamm Dis. 2017;5:480-92.

44. Pant K, Saraya A, Venugopal SK. Oxidative stress plays a key role in butyrate-mediated autophagy via Akt/mTOR pathway in hepatoma cells. Chem-Biol Interact. 2017;273:99-106.

45. Topping DL, Clifton PM. Short-chain fatty acids and human colonic function: roles of resistant starch and nonstarch polysaccharides. Physiol Rev. 2001;81:1031-64. 
46. Liu Q, Liu J, Roschmann KI, van Egmond D, Golebski K, Fokkens WJ, et al. Histone deacetylase inhibitors up-regulate LL-37 expression independent of toll-like receptor mediated signalling in airway epithelial cells. J Inflamm. 2013;10:15.

47. Kanai T, Mikami Y, Hayashi A. A breakthrough in probiotics: clostridium butyricum regulates gut homeostasis and antiinflammatory response in inflammatory bowel disease. J Gastroenterol. 2015;50:928-39.

48. Zhang J, Chen XY, Liu P, Zhao JB, Sun J, Guan WY, et al. Dietary clostridium butyricum induces a phased shift in fecal microbiota structure and increases the acetic acid-producing bacteria in a weaned piglet model. J Agr Food Chem. 2018;66:5157-66

49. Andrade-Oliveira V, Amano MT, Correa-Costa M, Castoldi A, Felizardo RJF, de Almeida DC, et al. Gut bacteria products prevent AKI induced by ischemia-reperfusion. J Am Soc Nephrol. 2015;26:1877-88.

50. Tholozan JL, Samain E, Grivet JP, Moletta R, Dubourguier HC, Albagnac G. Reductive carboxylation of propionate to butyrate in methanogenic ecosystems. Appl Environ Microbiol. 1988;54:441-5. 